\title{
Product of Partition Logics, Orthoalgebras and Automata
}

\author{
Anatolij DVUREČENSKIJ ${ }^{1}$, Karl SVOZIL ${ }^{2}$ \\ ${ }^{1}$ Mathematical Institute, Slovak Academy of Sciences, \\ Štefánikova 49, SK-814 73 Bratislava, Slovakia \\ e-mail: dvurecen@mau.savba.sk \\ ${ }^{2}$ Institute for Theoretical Physics, Technical University of Vienna \\ Wiedner Hauptstr. 8-10/136, A-1040 Vienna, Austria \\ e-mail: svozil@tph.tuwien.ac.at \\ received June 11, 1996
}

\begin{abstract}
We attempt to define a coupled system consisting of two partition logics and we introduce a product of partition logics. These partition logics have a close connection with Moore and Mealy type automata. We show how the coupled system of two automata is connected with the product of partition logics, and persent some illustrative examples.
\end{abstract}

\section{Introduction}

In the axiomatic approach to quantum mechanics, the event structure of a physical system is identified with a quantum logic [3] or an orthoalgebra [21, 9] while in the case of a classical mechanics with a Boolean algebra [20]. Assume that we have two independent physical systems with event structures $P$ and $Q$, and we wish to regard them as a coupled system. The event structure $L$ of this coupled system is usually called a tensor product of $P$ and $Q$ and we write $L=P \otimes Q$.

Tensor products in various approaches have been studied in $[21,1,17,7,11,15$, 16, 10, 26, 27]. A tensor product of orthoalgebras has been investigated by Foulis and Bennett in [8] via a universal mapping property, and a tensor product of an orthoalgebra and a Boolean algebra is given in [10].

Somewhat more general structures than orthoalgebras are quasi-orthoalgebras, for which a binary operation $\oplus$ is not, in general, associative. Very important examples of quasi-orthoalgebras, partition logics, have an intimate connection [6] with the investigation of the empirical propositional structure of Moore and Mealy type automata.

The aim of the present paper is to introduce a weaker form of a tensor product of partition logics, a product of partition logics, and to show its connection with automata. We show that this structure exists.

\section{Orthoalgebras}

The notion of orthoalgebras (or quasi-orthoalgebras) goes back to axiomatic models of quantum mechanics introduced by Foulis and Randall [11,21] as special algebraic

\footnotetext{
${ }^{1}$ The paper has been partially supported by grant G 229/94 SAV, Bratislava, Slovakia, and by the Mitteln zur Förderung der Auslandsbeziehungen an der Technischen Universität Wien. International Journal of Theoretical Physics, Vol 35, No. 11, 1966, 2409-2422.
} 
structures describing propositional logics.

A quasi-orthoalgebra is a set $L$ endowed with two special elements $0,1 \in L$ $(0 \neq 1)$ and equipped with a partially defined binary operation $\oplus$ satisfying the following conditions for all $a, b \in L$ :

(oai) if $a \oplus b$ is defined, then $b \oplus a$ is defined and $a \oplus b=b \oplus a$ (commutativity law);

(oaii) $a \oplus 0$ is defined for any $a \in L$ and $a \oplus 0=a$;

(oaiii) for any $a \in L$, there is an element $a^{\prime} \in L$ such that $a \oplus a^{\prime}$ is defined and $a \oplus a^{\prime}=1$ (orthocomplementation law);

(oaiv) if $a \oplus\left(a^{\prime} \oplus b\right)$ is defined, then $b=0$;

(oav) if $a \oplus(a \oplus b)$ is defined, then $a=0$;

(oavi) if $a \oplus b$ is defined, then $a \oplus(a \oplus b)^{\prime}$ is defined and $b^{\prime}=a \oplus(a \oplus b)^{\prime}$.

The following facts are true:

Proposition 2.1 Let $L$ be a quasi-orthoalgebra, $a, b \in L$. Then

(a) $0^{\prime}=1,1^{\prime}=0$

(b) $\left(a^{\prime}\right)^{\prime}=a$;

(c) if $a \oplus b=a \oplus c$, then $b=c$;

(d) if $a \oplus b=1$, then $b=a^{\prime}$.

The unique element $a^{\prime}$ is called the orthocomplement of $a \in L$, and the unary operation ' $^{\prime} L \rightarrow L$ defined by $a \mapsto a^{\prime}, a \in L$, is said to be an orthocomplementation. We shall say that two elements $a, b \in L$ (i) are orthogonal, and write $a \perp b$, iff $a \oplus b$ is defined in $L$ (it is clear that $a \perp b$ iff $b \perp a$ ), and (ii) $a \leq b$ iff there is an element $c \in L$ with $a \oplus c=b$.

It is easily shown that the relation $\leq$ is reflexive and antisymmetric, but need not be transitive. An associative quasi-orthoalgebra, i.e., a quasi-orthoalgebra, for which the associative law

(oavii) if $a \oplus b,(a \oplus b) \oplus c$ are defined in $L$, so are $b \oplus c$ and $a \oplus(b \oplus c)$, and $(a \oplus b) \oplus c=$ $a \oplus(b \oplus c)$

holds is said to be an orthoalgebra (OA in abbreviation). If in $L$ there are the elements $(a \oplus b) \oplus c$, and $a \oplus b(\oplus c)$ and if they coincide, we denote it as $a \oplus b \oplus c$. In any orthoalgebra, $\leq$ is transitive. On other hand, it is possible to give an example of a quasi-orthoalgebra with transitive $\leq$ which does not correspond to any orthoalgebra. If the elements $a \oplus(b \oplus c)$ and $(a \oplus b) \oplus c$ exist in a quasi-orthoalgebra and if they coincide, we denote them as $a \oplus b \oplus c$.

Due to Golfin [12], an orthoalgebra is a set $L$ with two special elements $0,1 \in L$ $(0 \neq 1)$ and endowed with a partial binary operation $\oplus$ satisfying (oai), (oaii), (oaiii), (oavii), and (oav*) if $a \oplus a$ is defined, then $a=0$.

The original idea of the partial binary operation $\oplus$ goes back to Boole's pioneering work [2], where he wrote $a+b$ as the logical disjunction of events $a$ and $b$ when the logical conjuction $a b=0$, so that, for mutually excluding events $a$ and $b$, $a+b$ is defined. This is all that is needed for probability theory: if $a b=0$, then $P(a+b)=P(a)+P(b)$. To avoid confusion, we write $a \oplus b$ for $a+b$ when $a b=0$.

Note that one can rewrite axioms for a Boolean algebra in terms of Boole's ideas of $a+b$. For more details, see Foulis and Bennett [8].

In addition, let $L$ be an orthomodular poset (OMP for abbreviation) (or an orthomodular lattice, OML in short), i.e., a poset $L$ with the least and last elements 0 and 1 and a unary operation ${ }^{\perp}: L \rightarrow L$, called an orthocomplementation, such that, for all $a, b \in L$, 
(i) $\left(a^{\perp}\right)^{\perp}=a$;

(ii) if $a \leq b$, then $b^{\perp} \leq a^{\perp}$;

(iii) $a \vee a^{\perp}=1$;

(iv) if $a \leq b^{\perp}$ (and we write $a \perp b$ ), then $a \vee b \in L$;

(v) if $a \leq b$, then $b=a \vee\left(a \vee b^{\perp}\right)^{\perp}$.

(For OML, $L$ has to be additionally a lattice.) Then $L$ can be organized into an OA if the binary operation $\oplus$ is defined via $a \oplus b$ exists in $L$ iff $a \leq b^{\perp}$ and $a \oplus b:=a \vee b$. The unary operation ' $: L \rightarrow L$ is defined via $a^{\prime}:=a^{\perp}, a \in L$.

We recall that if $L$ is an $\mathrm{OA}$ and $a, b \in L$ are mutually orthogonal, then $a, b \leq$ $a \oplus b$, and $a \oplus b$ is the minimal upper bound for $a$ and $b$ (i.e., $a, b \leq a \oplus b$, and if there is $c \in L$ with $a, b \leq c \leq a \oplus b$, then $c=a \oplus b)$, but this does not mean that $a \vee b$ exists in $L$, so that $L$ cannot necessarily be an OMP.

A subset $A$ of a quasi-OA (OA) $L$ is a quasi-suborthoalgebra (suborthoalgebra) of $L$ is (i) $0,1 \in A$; (ii) if $a \in A$, then $a^{\prime} \in A$; (iii) $a, b \in A$ with $a \perp b$ implies $a \oplus b \in L$.

If a (quasi)-suborthoalgebra $A$ of $L$ is, in addition, a Boolean algebra with respect to $\leq, A$ is called a Boolean suborthoalgebra of $L$. Denote by $\vee_{A}$ and $\wedge_{A}$ the join and the meet taken only in $A$, respectively. Then, $a \oplus b=a \vee_{A} b$ whenever $a, b \in A$ and $L$ is an OA. A maximal Boolean suborthoalgebra of $L$ is called a block.

\section{Partition Logics}

In this section, we present a notion of partition logics which will have an intimate connection with special types of automata, and which will generalize the results of Svozil [25] and Schaller and Svozil [22, 23, 24].

Let $L$ be a quasi-orthoalgebra with $\leq$. A non-void subset $I$ of $L$ is said to be an ideal of $L$ if

(i) if $a \in I, b \in L, b \leq a$, then $b \in I$;

(ii) $a, b \in I$ with $a \perp b$ imply $a \oplus b \in I$.

It is clear that $0 \in I$. An ideal $I$ of $L$ is said to be (i) proper if $I \neq L$ or, equivalently, $1 \notin I$; (ii) prime if, for any $a \in L$, either $a \in I$ or $a^{\prime} \in I$. We denote by $P(L)$ the set of all prime ideals in $L$.

A probability measure (or also a state) on $L$ is a mapping $s: L \rightarrow[0,1]$ such that (i) $s(1)=1$, and (ii) $s(a \oplus b)=s(a)+s(b)$ whenever $a \perp b$. A probability measure $s$ is two-valued if $s(a) \in\{0,1\}$ for any $a \in L$.

We recall that there is a one-to-one correspondence between two-valued probability measures and prime ideals: If $s$ is a two-valued probability measure, then $I_{s}=\{a \in L: s(a)=0\}$ is a prime ideal; and if $I$ is a prime ideal, then $s_{I}: L \rightarrow[0,1]$ defined via $s_{I}(a)=0$ iff $a \in I$, otherwise $s_{I}(a)=1$, is a two-valued probability measure on $L$.

A set $\mathcal{S}$ of probability measures on $L$ is called separating if for all $a, b \in L, a \neq b$, there is a probability measure $s \in \mathcal{S}$ such that $s(a) \neq s(b) . L$ is called prime iff it has a separating set of two-valued probability measure or, equivalently, for any different elements $a, b \in L$ there is a prime ideal $I$ of $L$ such that $a \in I$ and $b \notin I$.

Let $\mathcal{L}$ be a family of quasi-orthoalgebras (or OAs, OMPs, Boolean algebras, etc.) satisfying the following conditions: For all $P, Q \in \mathcal{L}, P \cap Q$ is a quasisuborthoalgebra (subOA, sub OMP, Boolean subalgebra, etc.) of both $P$ and $Q$, and the partial orderings and orthocomplementations coincide on $P \cap Q$. Define the set $L=\bigcup:=\bigcup\{P: P \in \mathcal{L}\}$, a relation $\oplus$, and the unary operation ' as follows:

(i) $a \oplus b$ iff there is a $P \in \mathcal{L}$ such that $a, b \in P$ and $a \perp_{P} b$, then $a \oplus b=a \oplus_{P} b$;

(ii) $a^{\prime}=b$ iff there is a $P \in \mathcal{P}$ such that $a, b \in P$ and $a^{\prime}=b$. 


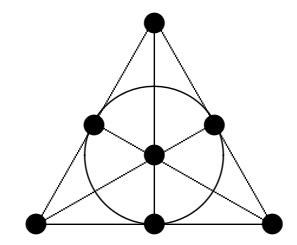

Figure

Figure 1: The Fano plane.

The set $L$ with the above defined $\oplus$ is called the pasting of the family $\mathcal{L}$.

Let $\mathcal{R}$ be a family of finite partitions of a fixed set $X$. The pasting of the family of Boolean algebras $\left\{B_{R}: R \in \mathcal{R}\right\}$ is called partition logic, and we denote it as a couple $(X, \mathcal{R})$.

We note that, for $a, b \in L=(X, \mathcal{R}), a \oplus b$ is defined on $L$ iff there exists a decomposition $R \in \mathcal{R}$ such that $a, b \in B(R)$ and $a \cap b=\emptyset$, where $B(R)$ is a Boolean algebra generated by $R$; then $a \oplus b:=a \cup b$.

We recall that two quasi-orthoalgebras $L_{1}$ and $L_{2}$ are isomorphic iff there is a one-to-one mapping $\phi: L_{1} \rightarrow L_{2}$ such that $a \oplus b$ is defined in $L_{1}$ iff $\phi(a) \oplus \phi(b)$ is defined in $L_{2}$ and $\phi(a \oplus b)=\phi(a) \oplus \phi(b)$.

The following result [6] describes quasi-orthoalgebras isomorphic to partition logics.

Theorem 3.1 A quasi-orthoalgebra $L$ is isomorphic to a partition logic if and only if $L$ is prime.

It is worth noting that if in a prime quasi-orthoalgebra $L$ there exist elements $x:=(a \oplus b) \oplus c$ and $y:=a \oplus(b \oplus c)$, for $a, b, c \in L$, then $x=y$. Indeed, by Theorem 3.1, there is a separating system of two-valued probability measures on $L$, $\mathcal{S}$, and a probability measure $s \in \mathcal{S}$ such that $s(x) \neq s(y)$. Then $s(a \oplus b)+s(c) \neq$ $s(a)+s(b \oplus c)$, which gives the contradiction $s(a)+s(b)+s(c) \neq s(a)+s(b)+s(c)$.

In any rate, the existence of one of $x$ or $y$ in $L$ does not imply the existence of the second one in $L$; see Example 4.1 below.

\section{Coupled Systems of Partition Logics}

The tensor product of orthoalgebras in the category of orthoalgebras has been studied by Foulis and Bennett [8]. They showed that if both orthoalgebras $P$ and $Q$ have "enough" probability measures, then the tensor product of $P$ and $Q$ exists. However, they have found an example of an orthoalgebra, the Fano plane drawn in Fig . 1, for which the tensor product $P \otimes P$ fails in the category of orthoalgebras. Dvurečenskij [4] showed that if we use a more general structure, effect algebras, then the tensor product of the Fano plane with itself exists in the category of effect algebras. In general, if both effect algebras $P$ and $Q$ have a non-empty system of probability measures, then the tensor product $P \otimes Q$ exists in the category of effect algebras.

Partition logics have a separating system of two-valued probability measures, unfortunately, they are not, in general, effect algebras, because they are not even (associative) orthoalgebras. Therefore, for the tensor product of partition logics we have to look for another way how to introduce it. 


\subsection{Product of Partition Logics}

Assume that $(X, \mathcal{R})$ and $(Y, \mathcal{S})$ are two partition logics, where $\mathcal{R}$ and $\mathcal{S}$ are two systems of finite decompositions of $X$ and $Y$, respectively. Choose $R \in \mathcal{R}$ and $S \in \mathcal{S}$. Then $R \times S:=\{A \times B: A \in R, B \in S\}$ is a decomposition of $X \times Y$. Define

$$
\mathcal{R} \times \mathcal{S}:=\{R \times S: R \in \mathcal{R}, S \in \mathcal{S}\} .
$$

Then the partition logic $\{X \times Y, \mathcal{R} \times \mathcal{S}\}$ is called the product partition logic of $(X, \mathcal{R})$ and $(Y, \mathcal{S})$.

Example 4.1 Let $\Omega=\{1,2,3,4,5,6\}$ and let $B_{1}$ and $B_{2}$ be the Boolean algebras generated by $R_{1}:=\{\{1\},\{2\},\{3\},\{4\},\{5,6\}\}$ and $R_{2}:=\{\{1\},\{2\},\{3,4\},\{5\},\{6\}\}$, respectively. The system $\left(\Omega,\left\{R_{1}, R_{2}\right\}\right)$ is a partition logic which is not a Boolean algebra, it is only a quasi-orthoalgebra. Then $\left(\Omega \times \Omega,\left\{R_{1} \times R_{1}, R_{1} \times R_{2}, R_{2} \times\right.\right.$ $\left.\left.R_{1}, R_{2} \times R_{2}\right\}\right)$ is a product partition logic of $\left(\Omega,\left\{R_{1}, R_{2}\right\}\right)$ with itself.

Proposition 4.2 Let $\mu$ and $\nu$ be probability measures on the partition logics $(X, \mathcal{R})$ and $(Y, \mathcal{S})$, respectively, where card $(Y)=n$ and $\mathcal{S}=\left\{\left\{y_{1}\right\}, \ldots,\left\{y_{n}\right\}\right\}, y_{i} \in Y$ for $i=1, \ldots, n, y_{i} \neq y_{j}$ for $i \neq j$, and $n \geq 1$. Then there is a unique probability measure $\mu \times \nu$ on $(X \times Y, \mathcal{R} \times \mathcal{S})$ such that

$$
(\mu \times \nu)(A \times B)=\mu(A) \nu(B), A \in(X, \mathcal{R}), B \in(Y, \mathcal{S}) .
$$

If, in addition, $\mu$ and $\nu$ are two-valued measures, so is $\mu \times \nu$.

Proof. Let $U$ be any element of the product partition logic $L=(X \times Y, \mathcal{R} \times \mathcal{S})$. Without loss of generality we can suppose that $Y=\{1, \ldots, n\}$. It is easy to show that $U$ can be represented uniquely in the form $U=\bigcup_{i=1}^{n} A_{i} \times\{i\}$, where all $A_{i}$ 's belong to the same Boolean algebra $B_{R}$ generated by a decomposition $R \in \mathcal{R}$; the case $A_{i}=\emptyset$ is not excluded.

In addition, $U:=\bigcup_{i=1}^{n} A_{i} \times\{i\} \perp \bigcup_{i=1}^{n} B_{i} \times\{i\}=: V$ iff $\left\{A_{i}\right\}_{i=1}^{n}$ and $\left\{B_{i}\right\}_{i=1}^{n}$ belong to the same Boolean algebra $B_{R}$ and $A_{i} \cap B_{i}=\emptyset$ for $i=1, \ldots, n$, and in this case $\bigcup_{i=1}^{n} A_{i} \times\{i\} \oplus \bigcup_{i=1}^{n} B_{i} \times\{i\}=\bigcup_{i=1}^{n}\left(A_{i} \cup B_{i}\right) \times\{i\}=\bigcup_{i=1}^{n}\left(A_{i} \oplus B_{i}\right) \times\{i\}$.

If now we define $(\mu \times \nu)(U):=\sum_{i=1}^{n} \mu\left(A_{i}\right) \nu(\{i\})$, then $\mu \times \nu$ is a well-defined mapping on $L$. It is simple to verify that $X \times Y=\bigcup_{i=1}^{n} X \times\{i\}$. Hence $(\mu \times \nu)(1)=$ $(\mu \times \nu)(X \times Y)=\sum_{i=1}^{n} \mu(X) \nu(\{i\})=\mu(X) \nu(Y)=1$.

Similarly, $(\mu \times \nu)(U \oplus V)=(\mu \times \nu)\left(\bigcup_{i=1}^{n}\left(A_{i} \oplus B_{i}\right) \times\{i\}\right)=\sum_{i=1}^{n} \mu\left(A_{i} \oplus\right.$ $\left.B_{i}\right) \nu(\{i\})=\sum_{i=1}^{n}\left(\mu\left(A_{i}\right)+\mu\left(B_{i}\right)\right) \nu(\{i\})=(\mu \times \nu)(U)+(\mu \times \nu)(V)$, so that $\mu \times \nu$ is a probability measure on $L$ satisfying (1). The uniqueness of $\mu \times \nu$ is now evident.

The probability measure $\mu \times \nu$, if it exists, is said to be a product probability measure of $\mu$ and $\nu$. We note that the extension of $\mu \times \nu$ from (1) to a probability measure on the product of general partition logics seems to be open.

\subsection{Tensor Product of Prime Quasi-Orthoalgebras}

According to Theorem 3.1, any prime quasi-orthoalgebra is isomorphic to a partition logic. Therefore, the coupled system consisting of two prime orthoalgebras $P$ and $Q$ being again a prime quasi-orthoalgebra can be called a tensor product of $P$ and $Q$ and it is denoted as $P \otimes Q$. In addition, they have an intimate connection with the product of isomorphic partition logics. For a rigorous introduction of the tensor product we need the following definitions.

Let $P$ and $L$ be two quasi-orthoalgebras. A mapping $\phi: P \rightarrow L$ is said to be

(i) a morphism iff $\phi(1)=1$, and $p \perp q, p, q \in P$, implies $\phi(p) \perp \phi(q)$ and $\phi(p \oplus q)=\phi(p) \oplus \phi(q) ;$ 
(ii) a monomorphism iff $\phi$ is a morphism and $\phi(p) \perp \phi(q)$ iff $p \perp q$;

(iii) an isomorphism iff $\phi$ is a surjective monomorphism.

Let $P, Q, L$ be quasi-orthoalgebras. A mapping $\beta: P \times Q \rightarrow L$ is called a bimorphism iff

(i) $a, b \in P$ with $a \perp b, q \in Q$ imply $\beta(a, q) \perp \beta(b, q)$ and $\beta(a \oplus b, q)=\beta(a, q) \oplus$ $\beta(b, q)$;

(ii) $c, d \in Q$ with $c \perp d, p \in P$ imply $\beta(p, c) \perp \beta(p, d)$ and $\beta(p, c \oplus d)=\beta(p, c) \oplus$ $\beta(p, d)$

(iii) $\beta(1,1)=1$.

If $\beta: P \times Q \rightarrow L$ is a bimorphism, then $\beta(\cdot, 1): P \rightarrow L$ and $\beta(1, \cdot): Q \rightarrow L$ are morphisms. Therefore, for $p \in P$ and $q \in Q$, we have $\beta(p, 1)^{\perp}=\beta\left(p^{\perp}, 1\right)$, $\beta(1, q)^{\perp}=\beta\left(1, q^{\perp}\right)$ and $\beta(p, 0)=\beta(0, q)=0$.

Also, if $a, b, p \in P$ and $c, d, q \in Q$, we have $a \leq b \Rightarrow \beta(a, q) \leq \beta(b, q)$ and $c \leq d \Rightarrow \beta(p, c) \leq \beta(p, d)$.

Definition 4.3 Let $P$ and $Q$ be prime quasi-orthoalgebras. We say that a pair $(T, \tau)$ consisting of a prime quasi-orthoalgebra $T$ and a bimorphism $\tau: P \times Q \rightarrow T$ is a tensor product of $P$ and $Q$ iff the following conditions are satisfied:

(i) If $L$ is a quasi-orthoalgebra and $\beta: P \times Q \rightarrow L$ is a bimorphism, there exists a morphism $\phi: T \rightarrow L$ such that $\beta=\phi \circ \tau$.

(ii) For every element of $t \in T$, there is a block $B$ of $T$ such that $t=\bigoplus_{i=1}^{n} \tau\left(p_{i}, q_{i}\right)$, where $\tau\left(p_{i}, q_{i}\right)$ belongs to the block $B$ for $i=1, \ldots, n$.

It is not hard to show that if a tensor product $(T, \tau)$ of $P$ and $Q$ exists, it is unique up to an isomorphism, i.e., if $(T, \tau)$ and $\left(T^{*}, \tau^{*}\right)$ are tensor products of D-posets $P$ and $Q$, then there is a unique isomorphism $\phi: T \rightarrow T^{*}$ such that $\phi(\tau(p, q))=\tau^{*}(p, q)$ for all $p \in P, q \in Q$. Unless confusion threatens, we usually refer to $P \otimes Q$ rather than to $(P \otimes Q, \otimes)$, as being a tensor product.

Unfortunately, the authors do not know the conditions under which the tensor product of prime quasi-orthoalgebras exists in the category of prime quasiorthoalgebras.

If both components $P$ and $Q$ are prime orthoalgebras, then the tensor product of $P$ and $Q$ exists in the category of orthoalgebras. This follows from Theorem 6.1 [8]. Indeed, if $p \in P$ and $q \in Q$ are two non-zero elements of the prime orthoalgebras $P$ and $Q$, then there exist two probability measures $\mu$ and $\nu$ on $P$ and $Q$ such that $\mu(p)=1=\nu(q)$ (if $p=1$ then $\mu(1)=1$ for any $\mu$, if $p \neq 1$ then $0 \neq p^{\perp} \neq 1$ and the primeness and the separateness of $P$ entail the existence of a two-valued probability measure $\mu$ with $0=\mu\left(p^{\perp}\right) \neq \mu(1)=1$ ). By Theorem 6.1 of Foulis and Bennett, this is a sufficient condition for the existence of the tensor product of $P$ and $Q$ in the category of orthoalgebras. In addition, for all probability measures $\mu$ and $\nu$ on $P$ and $Q$, respectively, the product measure $\mu \times \nu$, defined by $\mu \times \nu(p \otimes q)=\mu(p) \nu(q), p \in P, q \in Q$, exists. However, we do not know whether this tensor product also exists in the category of prime quasi-orthoalgebras.

It seems more hopeful consider the coupled system consisting of a prime quasiorthoalgebra and a Boolean algebra, called a bounded Boolean power of a prime quasi-orthoalgebra; see also similar problems in [4] for D-posets and [10] for orthoalgebras. The authors hope to present some results for these coupled systems in the future. 


\section{Realization by Automata}

The product of finite automata logics has an intuitive and rather simple realization: If the sets of states of two automata $M_{1}=\left(S_{1}, I_{1}, O_{1}, \delta_{1}, \lambda_{1}\right)$ and $M_{2}=$ $\left(S_{2}, I_{2}, O_{2}, \delta_{2}, \lambda_{2}\right)$ are mutually disjunct, then the automaton partition logic of the parallel decomposition $M_{1} \| M_{2}$ of the two automata is the product $\left\{S_{1} \times S_{2}, \mathcal{R}_{1} \times \mathcal{R}_{2}\right\}$ of the automaton partition logics $\left\{S_{1}, \mathcal{R}_{1}\right\}$ and $\left\{S_{2}, \mathcal{R}_{2}\right\}$ associated with $M_{1}$ and $M_{2}$, respectively. For a definition of the notation, see below.

\subsection{Moore and Mealy Automata}

A finite sequential machine or automaton is a device with the following properties [18, 14, 13]: (i) A finite set of inputs which can be applied in a sequential order; (ii) a finite set of internal configurations or states; (iii) a finite set of outputs; (iv) a setup such that the present internal configuration and input uniquely determine the next internal configuration and the output.

A Moore (Mealy) automaton is a quintuple $M=(S, I, O, \delta, \lambda)$, where

(i) $S$ is a finite (nonempty) set of states;

(ii) $I$ is a finite (nonempty) set of inputs;

(iii) $O$ is a finite (nonempty) set of outputs;

(iv) $\delta: S \times I \longrightarrow S$ is a computable transition function;

(v) $\lambda: S \longrightarrow O$ is a computable output function (Moore automaton);

(v') $\lambda: S \times O \longrightarrow O$ is a computable output function (Mealy automaton).

In what follows and if not mentioned otherwise, $s, i$, and $o$ stand for a particular internal state, input, and output, respectively. Moore (Mealy) machines are represented by flow tables and state graphs.

Example 5.1 To illustrate this, assume a Mealy machine $M_{s}=(S, I, O, \delta, \lambda)$ which has $n$ states, $n$ inputs, and 2 outputs. That is

$$
\begin{aligned}
S & =\{1,2, \ldots, n\} \\
I & =\{1,2, \ldots, n\} \\
O & =\{0,1\} .
\end{aligned}
$$

Its transition and output functions are ( $\delta_{s, x}$ stands for the Kronecker delta function)

$$
\begin{aligned}
& \delta(s, i)=i, \\
& \lambda(s, i)=\delta_{s, i}= \begin{cases}1 & \text { if } s=i \\
0 & \text { if } s \neq i\end{cases}
\end{aligned}
$$

The flow table and state graph (for $n=3$ ) of this Mealy automaton is given in Fig. 2.

\subsection{Machine Isomorphism, Serial and Parallel Decomposi- tions}

Two automata $M_{1}=\left(S_{1}, I_{1}, O_{1}, \delta_{1}, \lambda_{1}\right)$ and $M_{2}=\left(S_{2}, I_{2}, O_{2}, \delta_{2}, \lambda_{2}\right)$ of the same type are isomorphic if and only if there exist three one-to-one mappings $f: S_{1} \longrightarrow$ $S_{2}, g: I_{1} \longrightarrow I_{2}, h: O_{1} \longrightarrow O_{2}$ such that $f\left[\delta_{1}\left(s_{1}, i_{1}\right)\right]=\delta_{2}\left[f\left(s_{1}\right), g\left(i_{1}\right)\right]$ and 


$M_{s}=$\begin{tabular}{|c|cccc||cccc|}
\hline $\mathrm{s} / \mathrm{i}$ & 1 & 2 & $\cdots$ & $\mathrm{n}$ & 1 & 2 & $\cdots$ & $\mathrm{n}$ \\
\hline 1 & 1 & 2 & $\cdots$ & $\mathrm{n}$ & 1 & 0 & $\cdots$ & 0 \\
2 & 1 & 2 & $\cdots$ & $\mathrm{n}$ & 0 & 1 & $\cdots$ & 0 \\
$\vdots$ & 1 & 2 & $\cdots$ & $\mathrm{n}$ & 0 & 0 & $\cdots$ & 0 \\
$\mathrm{n}$ & 1 & 2 & $\cdots$ & $\mathrm{n}$ & 0 & 0 & $\cdots$ & 1 \\
\hline
\end{tabular}

(a)

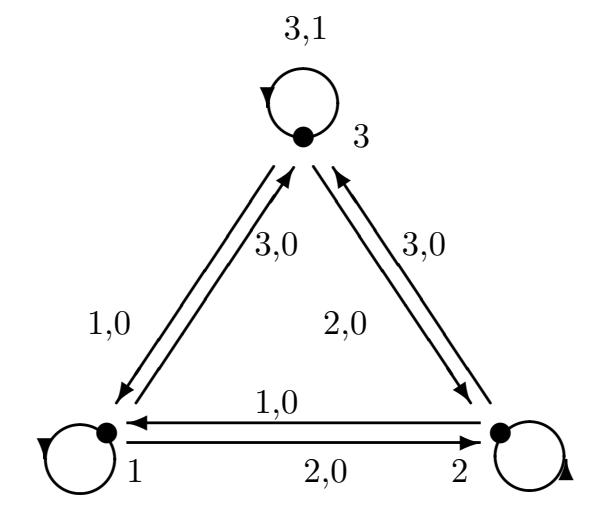

1,1

Figure 2:

$f\left[\lambda_{1}\left(s_{1}, i_{1}\right)\right]=\lambda_{2}\left[f\left(s_{1}\right), g\left(i_{1}\right)\right]$, where $s_{j} \in S_{j}$ and $i_{j} \in I_{j}, j \in\{1,2\}$. The triple $(f, g, h)$ is an isomorphism between $M_{1}$ and $M_{2}$. An isomorphism just renames the states, the inputs and the outputs. ¿From a purely input/output point of view, $g$ as well as $h\left(\right.$ or $\left.h^{-1}\right)$ are combinatory circuits and $M_{1}$ performs similarly to the serial decomposition (see below) $h^{-1} M_{2} g$ of the machines $g, M_{2}$ and $h^{1}$.

The serial connection of the two machines $M_{1}=\left(S_{1}, I_{1}, O_{1}, \delta_{1}, \lambda_{1}\right)$ and $M_{2}=$ $\left(S_{2}, I_{2}, O_{2}, \delta_{2}, \lambda_{2}\right)$ for which $O_{1}=I_{2}$ is the machine [13, p. 42]

$$
M=M_{1} \rightarrow M_{2}=\left(S_{1} \times S_{2}, I_{1}, O_{2}, \delta, \lambda\right)
$$

where $\delta\left[\left(s_{1}, s_{2}\right), i\right]=\left(\delta_{1}\left(s_{1}, i\right), \delta_{2}\left[s_{2}, \lambda\left(s_{1}, i\right)\right]\right)$ and $\lambda\left[\left(s_{1}, s_{2}\right), i\right]=\lambda_{2}\left[s_{2}, \lambda_{1}(s, i)\right]$.

The parallel connection of the two machines $M_{1}=\left(S_{1}, I_{1}, O_{1}, \delta_{1}, \lambda_{1}\right)$ and $M_{2}=$ $\left(S_{2}, I_{2}, O_{2}, \delta_{2}, \lambda_{2}\right)$ is the machine [13, p. 48]

$$
M=M_{1} \| M_{2}=\left(S_{1} \times S_{2}, I_{1} \times I_{2}, O_{1} \times O_{2}, \delta, \lambda\right)
$$

where $\delta\left[\left(s_{1}, s_{2}\right),\left(i_{1}, i_{2}\right)\right]=\left(\delta_{1}\left(s_{1}, i_{1}\right), \delta_{2}\left(s_{2}, i_{2}\right)\right)$ and $\lambda\left[\left(s_{1}, s_{2}\right),\left(i_{1}, i_{2}\right)\right]=\left(\lambda_{1}\left(s_{1}, i_{1}\right)\right.$, $\left.\lambda_{2}\left(s_{2}, i_{2}\right)\right)$.

The logical structure of the initial-state identification problem can be defined as follows. Let us call a proposition concerning the initial state of the machine experimentally decidable if there is an experiment $E$ which determines the truth value of that proposition. This can be done by performing $E$, i.e., by the input of a sequence of input symbols $i_{1}, i_{2}, i_{3}, \ldots, i_{n}$ associated with $E$, and by observing the output sequence

$$
\lambda_{E}(s)=\lambda\left(s, i_{1}\right), \lambda\left(\delta\left(q, i_{1}\right), i_{2}\right), \ldots, \lambda(\underbrace{\delta\left(\cdots \delta\left(q, i_{1}\right) \cdots, i_{n-1}\right)}_{n-1 \text { times }}, i_{n}) .
$$

The most general form of a prediction concerning the initial state $s$ of the machine is that the initial state $s$ is contained in a subset $P$ of the state set $S$. Therefore, we 


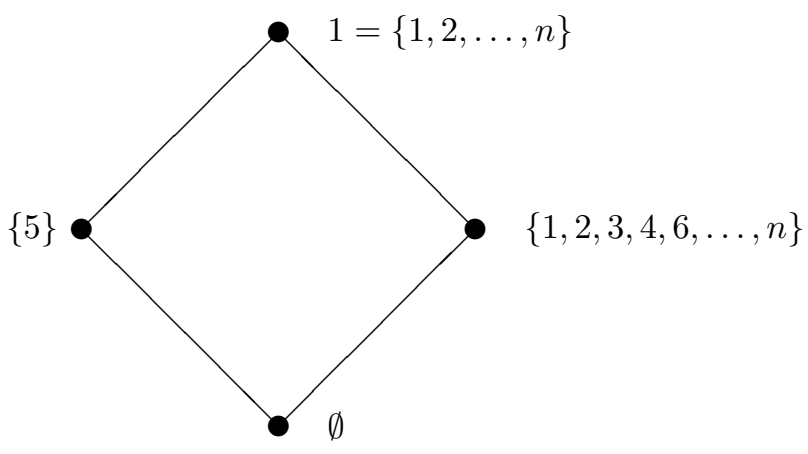

Figure 3:

may identify propositions concerning the initial state with subsets of $S$. A subset $P$ of $S$ is then identified with the proposition that the initial state is contained in $P$.

Let $E$ be an experiment (a preset or adaptive one), and let $\lambda_{E}(s)$ denote the obtained output of an initial state $s$. $\lambda_{E}$ defines a mapping of $S$ to the set of output sequences $O^{*}$. We define an equivalence relation on the state set $S$ by

$$
s \stackrel{E}{\equiv} t \text { iff } \lambda_{E}(s)=\lambda_{E}(t)
$$

for any $s, t \in S$. We denote the partition of $S$ corresponding to $\stackrel{E}{=}$ by $S / \stackrel{E}{\equiv}$. Obviously, the propositions decidable by the experiment $E$ are the elements of the Boolean algebra generated by $S / \stackrel{E}{\equiv}$, denoted by $B_{E}$.

Let $\mathcal{R}$ be the set of all Boolean algebras $B_{E}$. We call the partition logic $(S, \mathcal{R})$ an automaton propositional calculus.

Example 5.2 In what follows, we explicitly construct the Mealy automaton $M_{s}$ introduced before. Input/output experiments can be performed by the input of one symbol $i$ (in this example, more inputs yield no finer partitions). Let us assume that one input $i=5$. This experiment is able to distinguish between state $s=5$ and all the other states; hence it induces a partition (suppose $n>5$ )

$$
v(5)=\{\{5\},\{1,2,3,4,6, \ldots, n\}\} .
$$

After this experiment, information about the initial state is lost (irreversible model). Now consider the partitions $v(i)$ of all possible experiments with one input $x$ (all of them non-co-measurable). Every one of them generates a Boolean algebra of events with two atoms; e.g., $v(5)$ generates a two-element Boolean algebra $2^{2}$ whose Hasse diagram is drawn in Fig. 3.

The automaton propositional calculus and the associated partition logic is the set of all partitions

$$
P=\{v(i) \mid i \in I\} .
$$

Lattice-theoretically, this amounts to a pasting [19] of all the $v(i)$ 's. In the specific example, the pasting is just the horizontal sum-only the least and greatest elements $\emptyset$ and $\{1,2, \ldots, n\}$ of each $2^{2}$ is identified - and one obtains a Chinese lantern lattice $M O_{n}$. 


\section{References}

[1] Aerts, A., and Daubechies, I. (1978). Physical justification for using tensor product to describe quantum systems as one joint system, Helvetica Physica Acta, 51, 661-675.

[2] G. Boole, (1854). An Investigation of the Laws of Thought, Macmillan, London (reprinted Dover Press, New York, 1967).

[3] Busch, P., Lahti, P.J., and Mittelstaedt, P. (1991) The Quantum Theory of Measurement, Lecture Notes in Physics, Springer-Verlag, Berlin, Heidelberg, New York, London, Budapest.

[4] Dvurečenskij, A. (1995). Tensor product of difference spaces, Transactions of the American Mathematical Society, 347, 1043-1057.

[5] Dvurečenskij, A., and Pulmannová, S., (1994). Tensor product of D-posets and D-test spaces, Reports on Mathematical Physics, 34, 251-275.

[6] Dvurečenskij, A., Pulmannová, S., and Svozil, K. (1995). Partition logics, orthoalgebras and automata, Helvetica Physica Acta, 68, 407-428.

[7] Foulis, D.J. (1989). Coupled physical systems, Foundations of Physics, 19, 905922.

[8] Foulis, D.J., and Bennett, M.K. (1993). Tensor products of orthoalgebras, Order, 10, 271-282.

[9] Foulis, D.J., Greechie, R.J., and Rüttimann, G.T. (1992). Filters and supports in orthoalgebras, International Journal of Theoretical Physics, 31, 787-807.

[10] Foulis, D., and Pták, P. (1995). On the tensor product of a Boolean algebra and an orthoalgebra, Czechoslovak Mathematical Journal, 45 (120), 117-126.

[11] Foulis, D., and Randall, C. (1981). Empirical logic and tensor products, In:Interpretations and Foundations of Quantum Theories, A. Neumann ed., Wissenschaftsverlag, Bibliographisches Institut, Mannheim, pp. 9-20.

[12] Golfin, A. (1987). Representations and products of Lattices, Ph.D. Thesis, University of Massachusetts, Amherst, Massachusetts.

[13] Hartmanis, J., and Stearns, R.E. (1966). Algebraic Structure Theory of Sequential Machines, Prentice Hall, Englewood Cliffs, New Jersey.

[14] Hopcroft, J.E., and Ullman, J.D. (1979). Introduction to Automata Theory, Languages, and Computation. Addison-Wesley, Reading, Massachusetts.

[15] Kläy, M., Randall, C., and Foulis, D. (1987). Tensor products and probability weights, International Journal of Theoretical Physics, 26, 199-219.

[16] Lock, R. (1990). The tensor product of generalized sample spaces which admit a unital set of dispersion-free weights, Foundations of Physics, 20, 477-498.

[17] Matolcsi, T. (1975). Tensor product of Hilbert lattices and free orthodistributive product of orthomodular lattices, Acta Scientarum Mathematicarum (Szeged), 37, 263-272.

[18] Moore, E.F. (1956). Gedanken-experiments on sequential machines, In: C. E. Shannon and J. McCarthy, eds., Automata Studies. Princeton University Press, Princeton, New Jersey. 
[19] Navara, M., and Rogalewicz, V. (1991). The pasting constructions for orthomodular posets. Mathematische Nachrichten, 154, 157-168.

[20] Pták, P., and S. Pulmannová, S. (1991). Orthomodular Structures as Quantum Logics, Kluwer, Dordrecht, Boston, London.

[21] Randall, C., and Foulis, D. (1981). Empirical statistics and tensor products. In: Interpretations and Foundations of Quantum Theory, H. Neumann, ed., vol 5, Wissenschaftsverlag, Bibliographisches Institut, Mannheim, Germany, pp. 2128.

[22] Schaller, M., and Svozil, K. (1994). Partition logics of automata, Il Nuovo Cimento, 109B, 167-176.

[23] Schaller, M., and Svozil, M. (1995). Automaton partition logic versus quantum logic, International Journal of Theoretical Physics, 34 (8), 1741-1750.

[24] Schaller,M., and Svozil, K. (1996). Automaton logic, International Journal of Theoretical Physics, 35 (to appear).

[25] Svozil, K. (1993). Randomness and Undecidability in Physics, World Scientific, Singapore.

[26] Wilce, A. (1990). Tensor product of frame manuals, International Journal of Theoretical Physics, 29, 805-814.

[27] Zecca, A. (1978). On the coupling of quantum logics, Journal of Mathematical Physics, 19, 1482-1485. 